\title{
The Brain Locus of Interaction between Number and Size: A Combined Functional Magnetic Resonance Imaging and Event-related Potential Study
}

\author{
Roi Cohen Kadosh ${ }^{1}$, Kathrin Cohen Kadosh ${ }^{1,2}$, David E. J. Linden ${ }^{2,3}$, \\ Wim Gevers ${ }^{4}$, Andrea Berger ${ }^{1}$, and Avishai Henik ${ }^{1}$
}

\begin{abstract}
Whether the human brain is equipped with a special neural substrate for numbers, or rather with a common neural substrate for processing of several types of magnitudes, has been the topic of a long-standing debate. The present study addressed this question by using functional magnetic resonance imaging (fMRI) and event-related potentials (ERPs) together with the size-congruity paradigm, a Stroop-like task in which numerical values and physical sizes were varied independently. In the fMRI experiment, a region-of-interest analysis of the primary motor cortex revealed interference effects in the hemisphere ipsilateral to the response hand, indicating that the stimulus-stimulus conflict between numerical and physical magnitude is not completely resolved until response initiation.
\end{abstract}

\section{INTRODUCTION}

It is largely accepted that numerical magnitudes are processed in the intraparietal sulcus (IPS) in the human brain (see Dehaene, Piazza, Pinel, \& Cohen, 2003, for a meta-analysis). However, the assumption of a unique neural substrate reserved exclusively for numbers has been the topic of a long-standing debate (Cohen Kadosh \& Henik, 2006a; Cohen Kadosh et al., 2005; Shuman \& Kanwisher, 2004; Fias, Lammertyn, Reynvoet, Dupont, \& Orban, 2003; Walsh, 2003; Simon, 1999; Spelke \& Dehaene, 1999; Schwarz \& Heinze, 1998). Behavioral evidence of the size congruity effect (SCE) suggests that numerical and physical magnitudes interact at some level of processing. In the size congruity paradigm, participants have to compare either the numerical value or the physical size of two stimuli that differ in both dimensions, while ignoring the irrelevant dimension (Cohen Kadosh, Henik, \& Rubinsten, 2007; Schwarz \& Ischebeck, 2003; Tzelgov, Meyer, \& Henik, 1992; Henik \&

\footnotetext{
${ }^{1}$ Ben-Gurion University of the Negev, Beer Sheva, Israel, ${ }^{2}$ Johann Wolfgang Goethe University, Frankfurt am Main, Germany, ${ }^{3}$ University of Wales, UK, ${ }^{4}$ Ghent University, Belgium
}

This result supports the assumption of distinct comparison mechanisms for physical size and numerical value. In the ERP experiment, the cognitive load was manipulated in order to probe the degree to which information processing is shared across cognitive systems. As in the fMRI experiment, we found that the stimulus-stimulus conflict between numerical and physical magnitude is not completely resolved until response initiation. However, such late interaction was found only in the low cognitive load condition. In contrast, in the high load condition, physical and numerical dimensions interacted only at the comparison stage. We concluded that the processing of magnitude can be subserved by shared or distinct neural substrates, depending on task requirements.
Tzelgov, 1982). The stimuli may be incongruent (the physically larger digit is numerically smaller, e.g., 2 4) or congruent (the physically larger digit is also numerically larger, e.g., 4 2), with incongruent pairs being slower to process (SCE).

\section{At What Processing Stage is the Conflict Resolved?}

According to the early interaction account (Figure 1A), the relevant and irrelevant dimensions are processed in parallel until the comparison stage, where the conflict occurs. That is, the incongruent information interferes with the comparison process. Once the conflict has been resolved, the appropriate response is selected and executed. In contrast, the late interaction account (Figure 1B) postulates that the interaction occurs at a later stage of processing, namely, during response initiation (e.g., response selection, motor preparation, or response execution). According to this account, the irrelevant and relevant dimensions are processed in parallel at the comparison stage, and the conflict arises only at one of the response stages (Schwarz \& Heinze, 1998). 


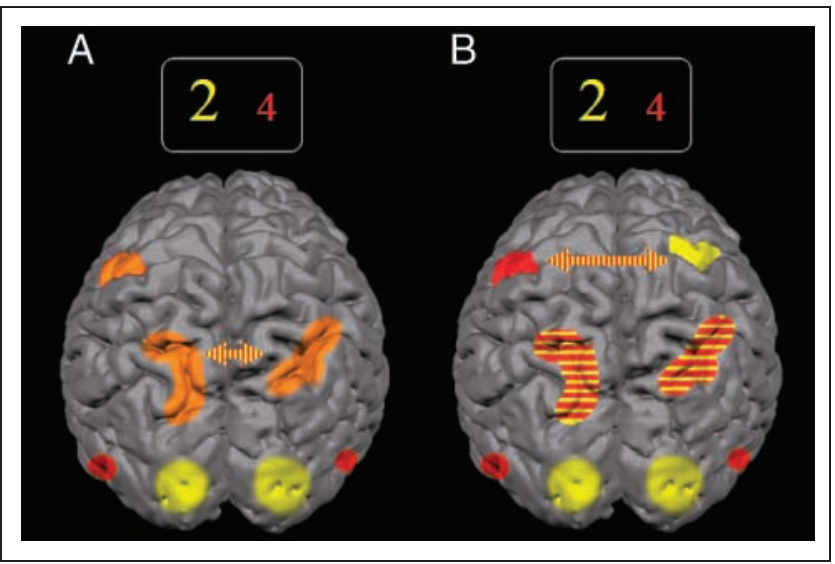

Figure 1. An anatomical illustration of early and late interactions. An anatomical illustration of the early (A) and late interaction (B) hypotheses during numerical comparison (where physical size has to be ignored). Numerical (red) and size (yellow) information are extracted in parallel by the occipital and occipito-temporal areas. (A) The comparison between the different stimuli is computed, and a common abstract representation for numerical value and physical size is produced in the IPS region (orange represents shared activation). The conflict between these dimensions occurs only at this stage (yellow-red arrow), and only later is the appropriate response selected, prepared, and executed in the motor cortex (orange). (B) The comparison of number (red) and physical size (yellow) is computed in parallel by separate neural substrates in the IPS. The two dimensions of the stimuli are processed up to the motor cortex, where response competition occurs (yellow-red arrow) and the overt response is produced according to the task requirements. (Connections between the two hemispheres are via callosal fibers).

\section{Empirical Evidence for Shared Magnitude Representation in the IPS}

Several neuroimaging and behavioral studies ascribed the interaction between the physical and the numerical dimensions to a shared representation of magnitude in the IPS (Cohen Kadosh \& Henik, 2006a, 2006b; Kaufmann et al., 2005; Pinel, Piazza, Le Bihan, \& Dehaene, 2004; Schwarz \& Heinze, 1998). Specifically, functional magnetic resonance imaging (fMRI) studies showed that the SCE modulates IPS activation (i.e., the incongruent condition yielded greater activation than the congruent condition; Kaufmann et al., 2005; Pinel et al., 2004). These studies seem to support the early interaction account. However, the overlap between brain networks, as identified by functional neuroimaging, should not be taken as direct evidence for a generic role of neurons in this brain area as subserving a common abstract representation of magnitude. This is because fMRI assesses activation in a given voxel that, in turn, reflects the activity of millions of neurons (Nieder, 2004). Moreover, the higher IPS activation for the incongruent condition in previous studies (Kaufmann et al., 2005; Pinel et al., 2004) does not necessarily rule out the late interaction account. Such higher activity for the incongruent condition (which includes irrelevant conflicting information), in contrast to the congruent condition, might be explained by one or more of the
Figure 2. Size congruity group results. Multisubject $(n=14)$ general linear model surface map, superimposed on flattened (middle panel) and inflated (top panel-medial view; bottom panel-lateral view) representations of the cortical sheet of a template brain. The orange color represents foci that showed significant activations for the main effect of size congruity. Activation was found over the bilateral posterior (Talairach coordinates, $x, y, z: 25,-65$, $38 ;-26,-55,44)$, and anterior IPS $(38,-44,45 ;-37,-37$, 43), frontal eye field (FEF) $(28,-12,58 ;-26,-10,57)$, anterior $(-5,5,41)$ and posterior $(2,-21,29)$ cingulate cortex (ACC/PCC), middle frontal gyrus (MFG) $(30,40$, $32 ;-28,40,32)$, inferior frontal gyrus (IFG) $(49,5,23$; $-47,1,28)$, frontal operculum (FOP) $(34,13,10 ;-35,5,10)$, occipito-temporal sulcus (OTS, shown only in the middle panel) $(34,-49,-12$;

$-38,-56,-10)$, and right presupplementary motor area $($ preSMA $)(5,7,51)$. CiS $=$ cingulate sulcus, IPS $=$ intraparietal sulcus, LS $=$ lateral sulcus, $\mathrm{SFS}=$ superior frontal gyrus, $\mathrm{RS}=$ Rolandic sulcus.

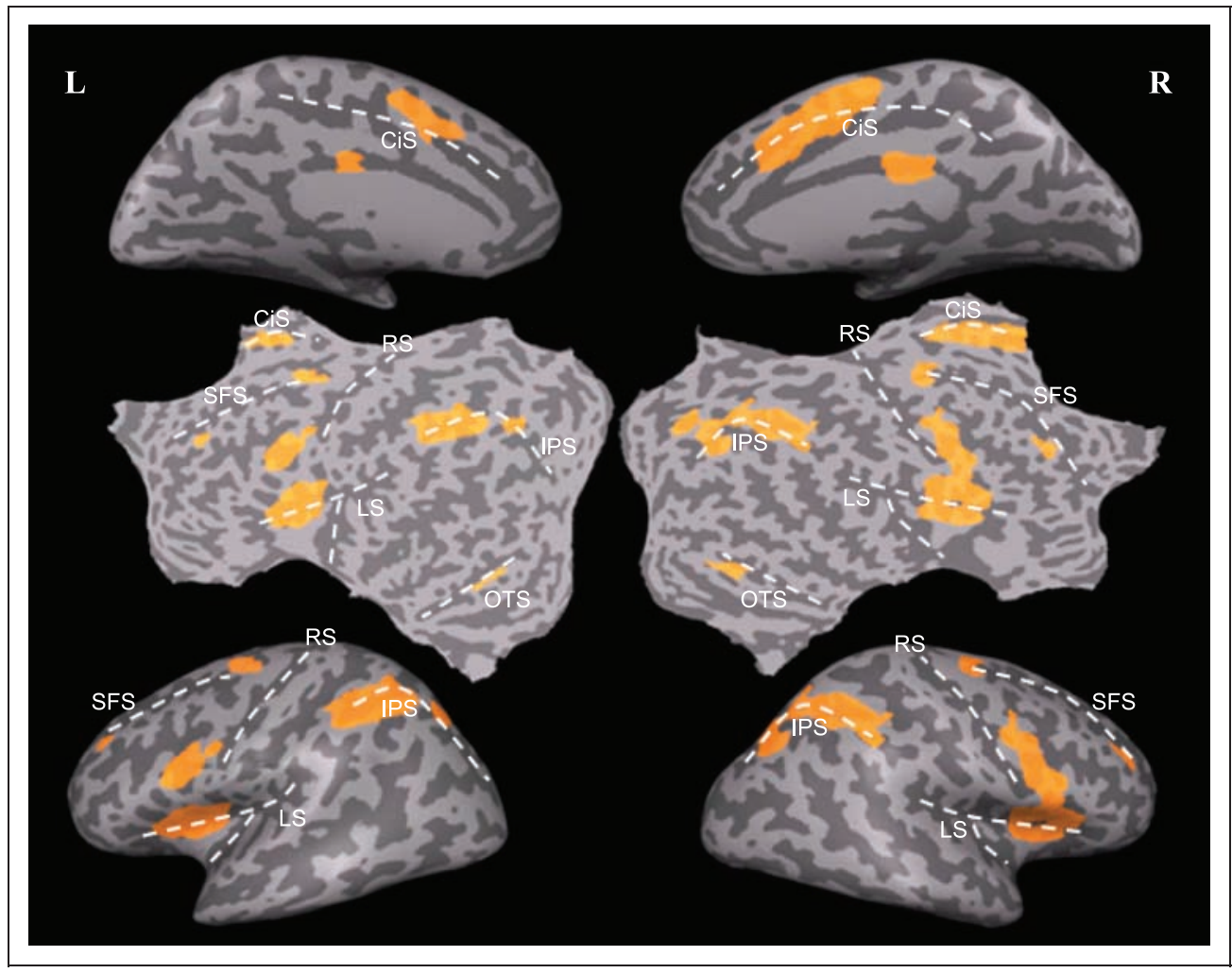


following alternatives: higher response selection demand (Göbel, Johansen-Berg, Behrens, \& Rushworth, 2004), higher demand on top-down attentional processes (Bledowski et al., 2004), or the necessity to suppress the task-irrelevant dimension (Wojciulik \& Kanwisher, 1999). Higher activation during incongruent than congruent conditions in imaging studies thus does not exclude the possibility that neurons in the IPS are activated solely by numerical information. Moreover, none of the previous neuroimaging studies that employed conflict tasks attempted to probe late interaction by an examination of motor cortex activation.

In contrast to fMRI, the event-related potential (ERP) technique offers a high temporal resolution that could be very helpful for investigating the exact time point of the conflict. By using this technique, Schwarz and Heinze (1998) showed that congruity conditions modulate changes in the morphology of the P300 component. The P300 is assumed to reflect top-down controlled stimulus categorization and evaluation (Kok, 2001), and some of its putative generators are located in the parietal lobe (Bledowski et al., 2004, 2006; Linden, 2005). These findings seem to support a common abstract representation of quantity and an early interaction account (Schwarz \& Heinze, 1998). However, there is a need to examine a possible late interaction between the numerical and physical dimensions directly. The lateralized readiness potential (LRP) (Gratton, Coles, Sirevaag, Eriksen, \& Donchin, 1988) is a suitable tool to investigate conflicts at the response stage. It is obtained by subtracting ipsilateral activity (nonresponse hand) from contralateral activity (response hand) (for a more detailed description, see the Methods section). According to the late interaction account, processing of the relevant and irrelevant dimensions will lead to concurrent activation of both motor cortices in incongruent conditions. Hence, the LRP of the incongruent condition will be different (that is, less negative) than that of the congruent condition. Schwarz and Heinze computed the LRP but did not find differences that support the late interaction account.

\section{The Current Study}

In order to test both the early and late interaction hypotheses, we used fMRI (Experiment 1) and ERP (Experiment 2). In Experiment 1, we examined the blood oxygenation level-dependent (BOLD) signal in the motor cortex, ipsilateral to the hand that was used for the actual response. This measurement has been termed lateralized BOLD response (LBR) (Dehaene et al., 1998), and is equivalent to the LRP (with ERP). Under the assumption of an early interaction, both dimensions will converge at the comparison stage, and higher activation for the incongruent relative to the congruent condition should be observed in the parietal lobe, but not in the motor cortex. Conversely, the late interac- tion model would predict higher motor activity for the incongruent versus congruent conditions because the different dimensions are processed in parallel up to the response initiation. The neural interference effect would mainly be expected in the hemisphere ipsilateral to the response hand, indicating that the irrelevant dimension succeeded in activating the corresponding motor system.

In Experiment 2, we further examined the possible coexistence of both shared and distinct comparison mechanisms by using ERP, motivated by the partly conflicting findings in the previous literature. One way to examine such bipartite comparison systems is to manipulate the cognitive load of a task (Lavie, 2005; Lavie, Hirst, de Fockert, \& Viding, 2004; Lavie \& Tsal, 1994). We hypothesized that increasing cognitive load (utilizing the distance effect) would lead to a shift from separate processing of physical and numerical magnitudes to reliance on overlapping resources. The conflict should then be resolved at the response stage under low cognitive load and at earlier stages under high cognitive load, as evidenced by LRP effects for low but not high cognitive load.

\section{fMRI EXPERIMENT}

\section{Results}

\section{Behavioral Results}

For every participant in each condition, the mean reaction time (RT) was calculated for correct trials only. These means were subjected to a two-way analysis of variance (ANOVA), with comparison and congruency as within-subject factors. RT and error rates confirmed that interference (i.e., incongruent vs. neutral and congruent), as indicated by a main effect for size congruity $[F(2,26)=118.40, p<.001]$, occurred for both tasks. The difference between incongruent (604 msec) versus neutral (525 msec) and congruent (486 msec) trials was significant $[F(1,13)=119.34, p<.001]$. Participants responded $64 \mathrm{msec}$ faster in the size comparison task $[F(1,13)=21.12, p<.001]$. The two-way interaction between task and congruity was not significant $(F<1$, $p>.2)$. For errors, only the main effect for congruency was significant $[F(2,26)=28.55, p<.001]$. Percentage of errors was larger for incongruent than neutral and congruent conditions $(2.7,0.18$, and 0.18 , respectively), and thus, excluded any RT-accuracy tradeoff.

\section{fMRI Results}

The behavioral interference resulted in higher activation in lateral prefrontal (middle frontal gyrus) and premotor (along the precentral sulcus, reaching into the inferior frontal gyrus) regions bilaterally and the medial prefrontal cortex. Most of these areas have also been 
reported in previous studies that utilized conflict situations (Kerns et al., 2004; Weissman, Warner, \& Woldorff, 2004; Stephan et al., 2003; Bush, Luu, \& Posner, 2000). In addition, interference correlated with higher activity in the IPS and occipito-temporal regions as in previous studies of the SCE (Kaufmann et al., 2005; Pinel et al., 2004) (Figure 2). In addition, the left frontal operculum showed a significant interaction between congruity and task (no congruity effect for the size comparison). No brain area showed the opposite pattern (higher activation for congruent or neutral vs. incongruent trials) in either comparison.

Most crucially, in the motor cortex (M1) region-ofinterest (ROI) analysis, we found main effects for congruity and for response hand $[F(2,26)=7.09, p<.005$, and $F(1,13)=51.44, p<.001$, respectively]. Moreover, the two-way interaction between congruity and response hand was significant $[F(2,26)=6.73, p<.005$; Figure 3]. There were no further main effects or interactions. Additional analyses of the two-way interaction were conducted separately for M1 ipsilateral and contralateral to the response hand. Activation in the primary motor cortex (M1) ipsilateral to the response hand showed an SCE in the absence of an overt response with the corresponding hand $[F(2,26)=10.26, p<$ $.001]$, indicating that the irrelevant dimension was processed up to the motor level (Figure 3). Additional analyses revealed a significant difference for the incongruent versus the neutral and congruent conditions $[F(1,13)=11.80, p<.005]$. However, M1 did not show a congruity effect for the contralateral hand $[F(2,26)=$ $2.37, p>1$. In order to exclude alternative explanations, we reanalyzed a dataset of tasks that involved easy versus difficult levels of comparative judgments published elsewhere (Cohen Kadosh et al., 2005). There was no effect for the ipsilateral hand as a function of speed of processing $[F \mathrm{~S}<1]$ even at a very liberal level of statistical threshold ( $p<.05$, uncorrected, fixed effect). Notably, in the current paradigm, this exclusively ipsilateral effect was not observed in any other interferencesensitive brain area. In addition, neither higher-order interactions nor the interaction between task, congruency, and response hand $[F<1, p>.4]$ was significant.

\section{Discussion}

fMRI activation both in the IPS and the primary motor cortex was modulated by the SCE. An ROI analysis of motor cortex activity revealed aberrant activation of the ipsilateral (to the response hand) motor cortex in the incongruent condition. This ipsilateral motor cortex activation indicates that the irrelevant dimension was processed up to the response selection stage. This M1
Figure 3. Motor cortex ROI analysis. (A) Areas of the motor cortex (in orange) that showed a significant interference effect for the nonresponse hand in absence of an overt response (Talairach coordinates: 38 , $-18,58,1671$ voxels, for the right hemisphere; Talairach coordinates: $-38,-20,53$, 1260 voxels, for the left hemisphere). (B) Beta weights of motor cortex activity for responses with the contralateral and ipsilateral hands in the conflict (red bars) or no-conflict (green bars) conditions. (C) The lateralized BOLD response (LBR) in the conflict (red line) and no-conflict (green line) conditions. Similar to the LRP (see Figure 7), the reduction in the percent signal change for the conflict condition is due to an additional activation for the ipsilateral hand. Error bars depict 1 SEM.

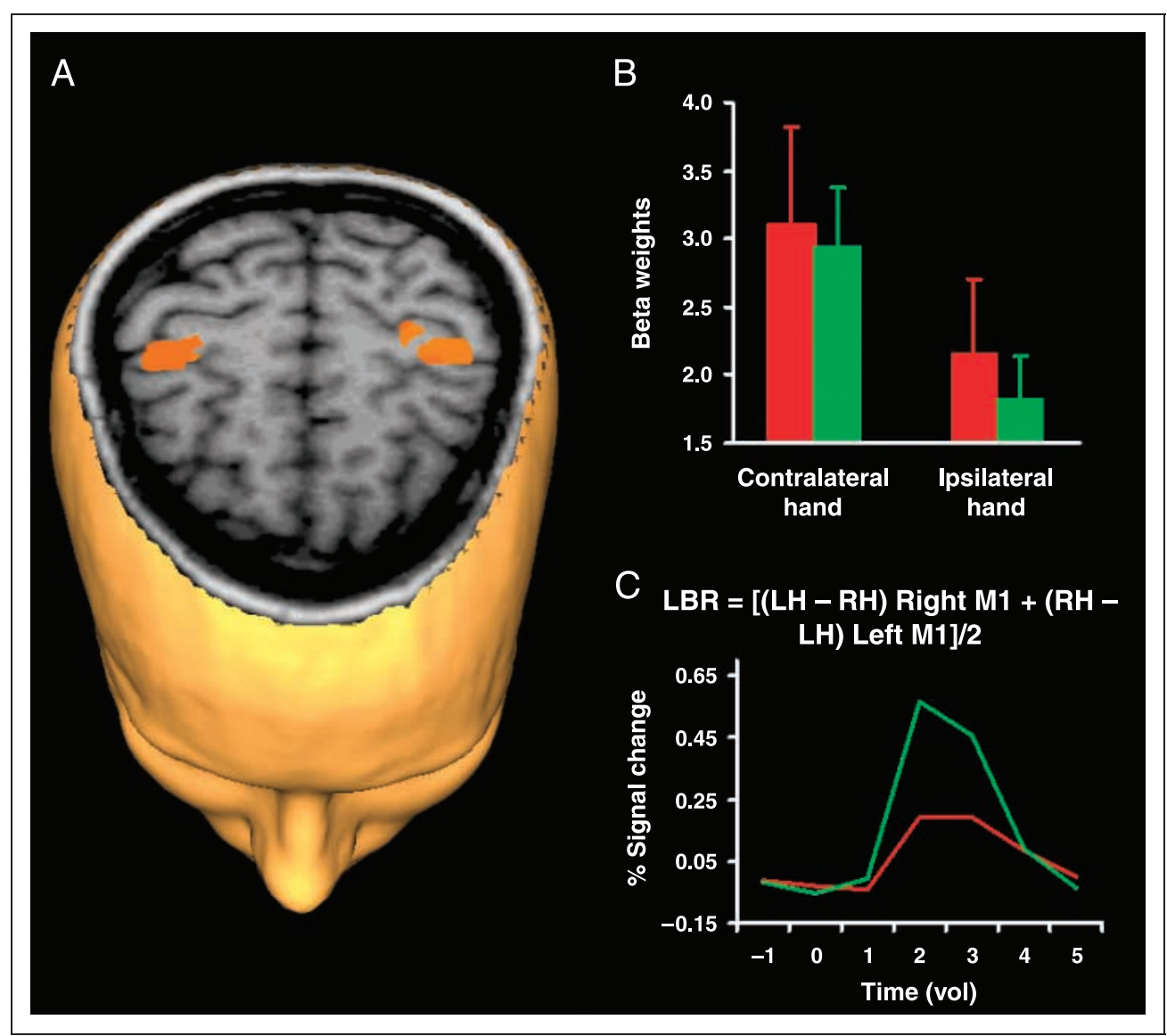


activation is hardly compatible with the idea that the human brain represents numbers, physical size, and other magnitudes in a single shared representation that involves the IPS (Cohen Kadosh \& Henik, 2006a, 2006b; Cohen Kadosh et al., 2005; Kaufmann et al., 2005; Fias et al., 2003; Schwarz \& Ischebeck, 2003; Walsh, 2003). However, the IPS modulation by the SCE contradicts the assumption that numerical and physical magnitudes have specialized representations in the parietal lobes (Spelke \& Dehaene, 1999) that can interact only at a later postcomparison stage in motor areas.

Hence, it is possible that both distinct and shared comparison systems exist. Such a bipartite comparison system assumption is in agreement with recent ideas about functional degeneracy in the human brain (Price \& Friston, 2002). Furthermore, it might help to explain why brain injury rarely produces a total inability to compare numbers.

One way to examine the possibility of distinct versus shared comparison systems is to manipulate the cognitive load of a task (Lavie, 2005; Lavie et al., 2004; Lavie \& Tsal, 1994). According to the load theory, both dimensions of a task can be processed in an automaticmandatory fashion, up to later stages of processing, as long as the cognitive capacity limit is not exceeded. This strategy of utilizing the effect of cognitive load to identify the stage of interference was mainly designed for perceptual tasks. However, it can also be extended to more cognitive or abstract tasks such as the current paradigm. In the case of the size congruity paradigm, it can be assumed that both dimensions can be processed until the response selection stage as long as capacity suffices for processing of both dimensions. If the capacity limit is exceeded, conflicts between magnitudes will be processed at the comparison stage and only one dimension will be processed throughout the (later) stage of response initiation.

\section{The Numerical Distance Effect as an Operationalization of Cognitive Load}

In the size congruity paradigm, cognitive load can be modulated by manipulating the numerical distance between the compared numbers. The numerical distance effect (Moyer \& Landauer, 1967) is indicated by an inverse relationship between numerical distance and responding time. For example, participants are faster to decide which digit is larger when comparing 2 and 8 than 2 and 4 . It has been suggested that digits that are closer in their numerical values have similar representations that overlap and, as a result, the comparison becomes more difficult. In the case of a large distance between two digits, representations are farther apart, thus allowing for faster comparison (Dehaene, 2003). Therefore, instead of manipulating the cognitive load in a perceptual task (Lavie, 2005; Lavie et al., 2004; Lavie \& Tsal, 1994), in the present study, the cognitive load is manipulated at the representational level. As in Experiment 1, previous studies that investigated the SCE did not examine the effect of load on the SCE (Pinel et al., 2004). Others did not differentiate between high and low load (Kaufmann et al., 2005), or examined only the effect of high load (i.e., 1 and 2 numerical distances) on the SCE, which might lead to spurious support for the early interaction account (Schwarz \& Heinze, 1998). Moreover, because numbers are processed in an automatic way (Schwarz \& Ischebeck, 2003; Dehaene \& Akhavein, 1995; Tzelgov et al., 1992; Henik \& Tzelgov, 1982), it would be beneficial to investigate the effect of load when it is task relevant (i.e., numerical distance in the numerical task), and when it is task irrelevant (i.e., numerical distance in the physical task).

\section{ERP EXPERIMENT}

In the current study, we investigated the SCE and its modulation in the ERP by using low load (a large numerical distance) and high load (a small numerical distance). In contrast to previous imaging studies that focused on the load effect of the relevant dimension (Schwartz et al., 2005; Rees, Frith, \& Lavie, 1997), we manipulated the load in the irrelevant dimension also.

Previous studies demonstrated that cognitive load can affect the processing stage (e.g., for a review, see Lavie, 2005). We hypothesized that manipulating cognitive load, in the current task, might affect the capacity of the strictly number-specific parts of the parietal cortex, and in the case of a high cognitive load, recruit areas that are also required for physical size comparison. Hence, load would affect the stages where an interaction between the irrelevant and relevant dimensions occurred. Moreover, we expected that changes in P300 and LRP morphology would help to pinpoint the stages of interaction between the dimensions of magnitude. The results from ERP would allow us to examine the observed effect in the fMRI with an improved temporal resolution.

\section{Results}

\section{Behavioral Results}

For every participant in each condition, mean RT was calculated for correct trials only. All RTs were between 200 and $1000 \mathrm{msec}$, hence, outlier cutoffs were not used. These means were subjected to a three-way ANOVA, with comparison, congruity, and cognitive load as withinsubject factors.

All main effects were significant. In addition, two twoway interactions were significant: comparison and cognitive load $[F(1,13)=83.56, p<.001]$, and comparison and congruity $[F(2,26)=15.22, p<.001]$. The threeway interaction between comparison, cognitive load, 
and congruity was also significant $[F(2,26)=32.57$, $p<.001]$. The three-way interaction is presented in Figure 4 . In order to disentangle the source of the threeway interaction, we conducted simple effects analyses for numerical and physical comparisons separately.

Numerical comparison. The simple interaction between congruity and cognitive load was significant $[F(2$, $26)=15.88, p<.001]$, and further analyses revealed an increase in the congruity effect (i.e., RT difference between incongruent and congruent conditions) as the cognitive load increased $[F(1,13)=23.03, p<.001]$, from $69 \mathrm{msec}$ for low cognitive load to $107 \mathrm{msec}$ for high cognitive load.

Physical comparison. The simple interaction between congruity and cognitive load was significant $[F(2,26)=$ $17.06, p<.001]$, and further analyses showed a decrease in the congruity effect as the cognitive load increased $[F(1,13)=20.34, p<.001]$, from 68 msec for low cognitive load to $38 \mathrm{msec}$ for high cognitive load.

Error rates. The three-way interaction between comparison, cognitive load, and congruity was significant $[F(2,26)=14.83, p<.001]$. The pattern of results was similar to that produced in the RT analysis. Thus, no time-accuracy tradeoff was found.

\section{ERP Results}

P300 amplitude. Visual inspection of the P300 activity on the scalp found the commonly reported centroparietal distribution (Rugg \& Coles, 1996). Statistical analysis focused on the Pz electrode. All main effects for P300 amplitude were significant: comparison effect $[F(1$, $13)=6.49, p<.005]$, cognitive load $[F(1,13)=5.72$, $p<.05]$, and congruity $[F(2,26)=18.67, p<.001]$. In addition, the interaction between comparison and cognitive load $[F(1,13)=15.72, p<.005]$ and the threeway interaction between comparison, cognitive load, and congruity $[F(2,26)=4.45, p<.05]$ were significant. It

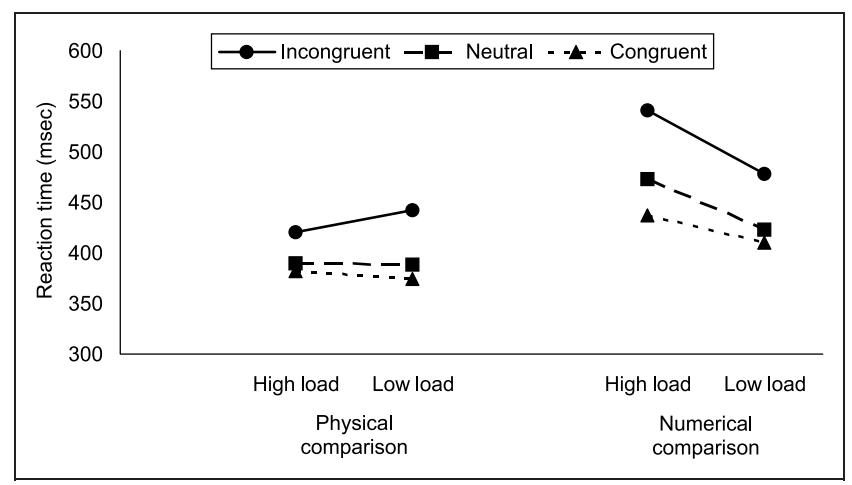

Figure 4. Reaction time as a function of comparison, cognitive, load and congruity. seems that the three-way interaction (Figures 5 and 6) mirrored the behavioral results. This visual inspection was further validated by a significant negative correlation between the P300 amplitude and RT $[r(12)=-.94, p<$ $.001]$. As in the behavioral data, to further our understanding regarding the source of the three-way interaction, we conducted simple effects analyses for numerical and physical comparisons separately.

Numerical comparison. Only the simple main effects for cognitive load and congruity were significant $[F(1,13)=19.61, p<.001$ and $F(2,26)=11.82, p<$ .001 , respectively], whereas the simple interaction between congruity and cognitive load was not significant $[F(2,26)=2.07, p=.14]$.

For cognitive load, we found a decrease in the P300 amplitudes as cognitive load increased (from 4.95 to $5.91 \mu \mathrm{V})$. The congruity effect was characterized by decreased P300 amplitudes in the incongruent condition $(4.47 \mu \mathrm{V})$ in comparison to the neutral and congruent conditions $(5.72$ and $6.11 \mu \mathrm{V}$, respectively) $[F(1,13)=$ $15.80, p<.001$ ] (Figure 5).

Physical comparison. Only the simple main effect for congruity was significant $[F(2,26)=7.43, p<.005]$, whereas the simple main effect for cognitive load and the simple interaction between congruity and cognitive load were not significant $[F<1$, and $F(2,26)=2.21$, $p=.13$, respectively] .

As in the numerical comparison task, the congruity effect was characterized by decreased P300 amplitudes in the incongruent condition $(5.43 \mu \mathrm{V})$ in comparison to the neutral and congruent conditions $(6.27$ and $6.92 \mu \mathrm{V}$, respectively) $[F(1,13)=11.94, p<.005]$ (Figure 6)

$L R P$ results. No significant differences were found between neutral and congruent conditions, as well as no interaction with any other factors. These findings gave statistical support for the assumptions made above (see Methods).

The only significant main effect was congruity $[F(1$, $13)=5.28, p<.05]$. In addition, only the interaction between cognitive load and congruity was marginally significant $[F(1,13)=3.46, p=.08]$, and the other interactions were not significant $(p s>.18)$. The interaction between cognitive load and congruity is presented in Figure 7. Because the effect of cognitive load was of major interest in this research, we further analyzed the data of the congruity condition for the two cognitive loads separately.

High cognitive load. Neither simple main effects nor simple interaction was significant [all $F<1$ ].

Low cognitive load. The simple main effect of comparison was not significant $[F(1,13)=1.94, n s]$. In 
Figure 5. Grand-averaged P300 waveforms recorded at electrode 68 for congruent, incongruent, and neutral trials at numerical comparison under high (A) and low (B) load.

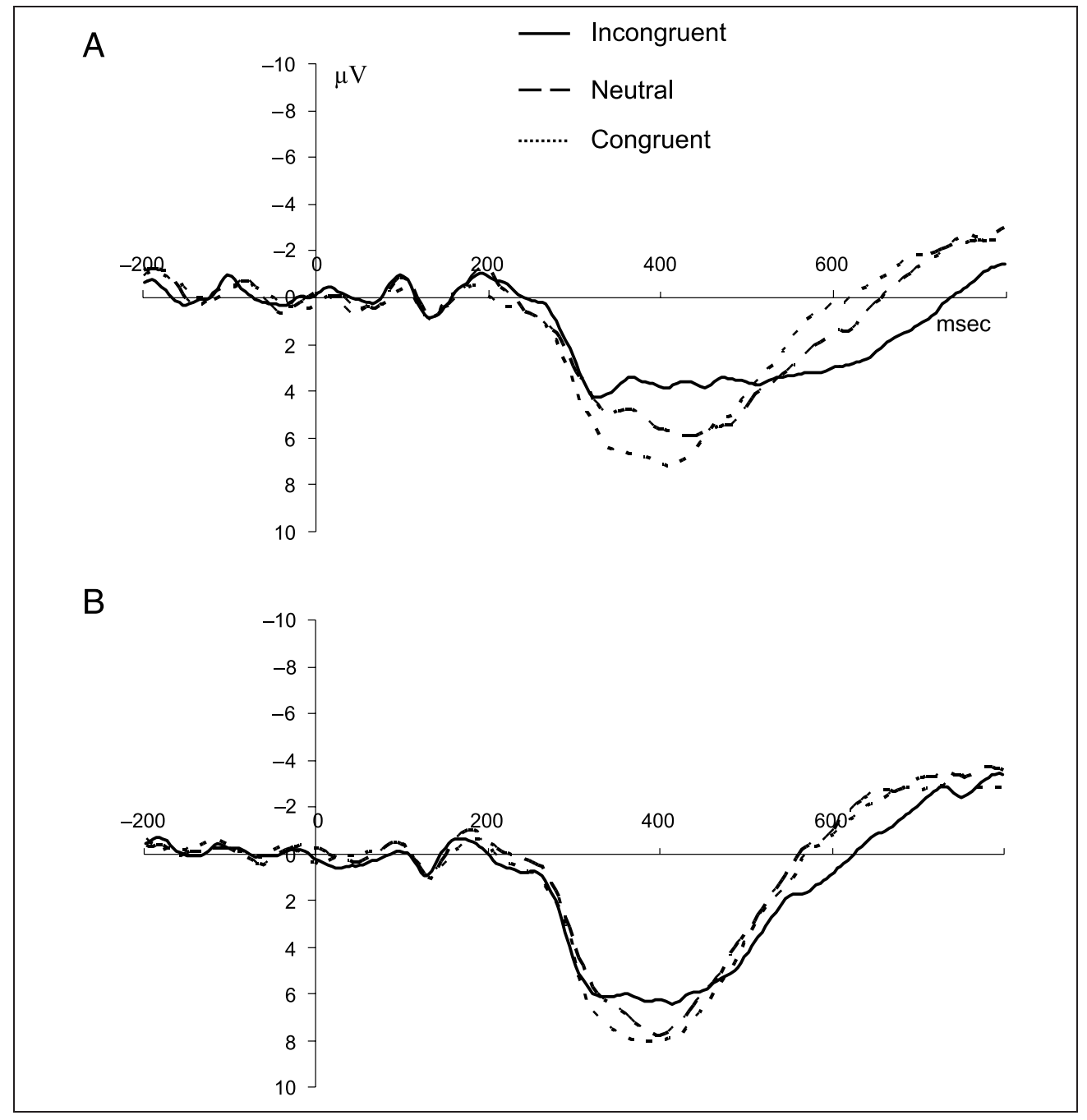

contrast, the simple main effect of congruity was significant $[F(1,13)=12.01, p<.005]$, with the incongruent condition being more positive $(0.3 \mu \mathrm{V})$ than the congruent and neutral conditions $(-0.42 \mu \mathrm{V})$. The simple interaction between congruity and comparison was not significant $[F(1,13)=1.43, n s]$.

\section{Discussion}

The current results shed new light on the two questions posed above. When is the conflict between the two interacting dimensions resolved? Are numbers processed by a distinct neural number system or a general magnitude system? Because the current task pits one magnitude dimension against the other, we expected that by resolving one question, we would answer the other as well.

At the behavioral level, we replicated the SCE for both numerical and physical comparisons. The size congruity was modulated by the cognitive load. However, the pattern of this interaction was different for the numerical and physical tasks. Namely, for the numerical task, the SCE increased as the (relevant) cognitive load increased. In contrast, in the physical task, the SCE decreased as the (irrelevant) cognitive load increased.

In the electrophysiological data, we observed a modulation of the P300 by the SCE, with reduced P300 amplitude for the incongruent condition. Interestingly, the P300 amplitude mirrored the behavioral results as indicated by a highly significant correlation between these two measures. Finally and most importantly, a clear LRP effect (i.e., an initial dip), indicating significant activation for the irrelevant information (incongruent vs. neutral and congruent conditions), was only obtained for the low cognitive load condition. These findings indicate that both dimensions are processed in parallel up to the response selection stage in the low, but not in the high, cognitive load condition.

\section{GENERAL DISCUSSION}

Earlier studies from our and other groups gave support for an early resolution of conflict at the comparison stage (Cohen Kadosh \& Henik, 2006a; Kaufmann et al., 
Figure 6. Grand-averaged P300 waveforms recorded at electrode 68 for congruent, incongruent, and neutral trials at physical comparison under high (A) and low (B) load.

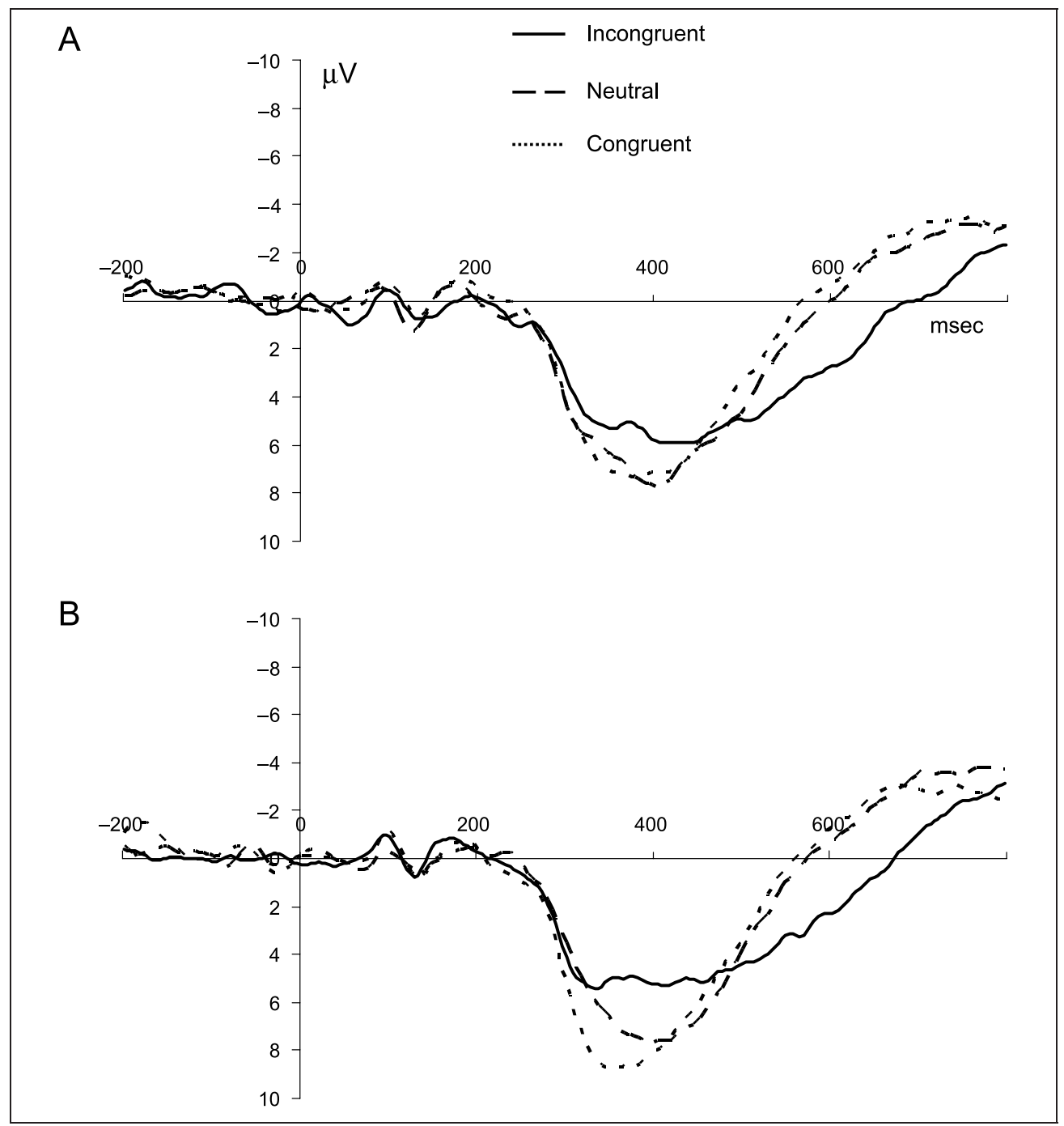

2005; Pinel et al., 2004; Schwarz \& Heinze, 1998). These results fit the suggestion of a shared comparison mechanism. However, our fMRI results (Experiment 1) support the notion of late resolution of conflict, which can fit with the suggestion that numbers are processed by a specific mechanism. Our ERP study (Experiment 2) manipulated physical sizes and numerical values independently as in previous studies. However, in order to disentangle the apparent contradiction between Experiment 1 and previous studies, and to investigate the possible coexistence of distinct and shared mechanisms, we also examined the effect of cognitive load on conflict resolution.

The current study shows that the locus of conflict resolution in the size congruity paradigm is not fixed, but can occur at both early and later stages of task processing, depending on specific experimental conditions. When the processing of both the relevant and irrelevant dimension is relatively easy (i.e., low cognitive load condition), both numerical value and physical size are processed in parallel up to the motor cortex. This observation does not necessarily indicate that the conflict is resolved at later motor-related stages. It does show, however, that the conflict between numerical size and physical value is not resolved by the time that the motor system is activated. This finding supports the hypothesis that the human brain has a specialized neuronal substrate for numerical processing (Spelke \& Dehaene, 1999). However, when the processing of the relevant and irrelevant dimensions is relatively difficult, the conflict between numerical value and physical size seems to be resolved already at the comparison stage. Such a bipartite comparison system of specialized and distinct comparison mechanisms is in agreement with recent ideas about functional degeneracy in the human brain (Price \& Friston, 2002), and it might help to explain why brain injury rarely produces a total inability to compare numbers.

What do these findings tell us about the specialization for numbers? The evidence from the low load condition 
Figure 7. Grand-averaged response-locked LRP for conflict (i.e., incongruent) and no-conflict (i.e., congruent and neutral) conditions under high (A) and low (B) load. Solid lines indicate conflict; dotted lines indicate no-conflict conditions. The black frame rectangle indicates the time window under which the interaction between load and congruity was computed.

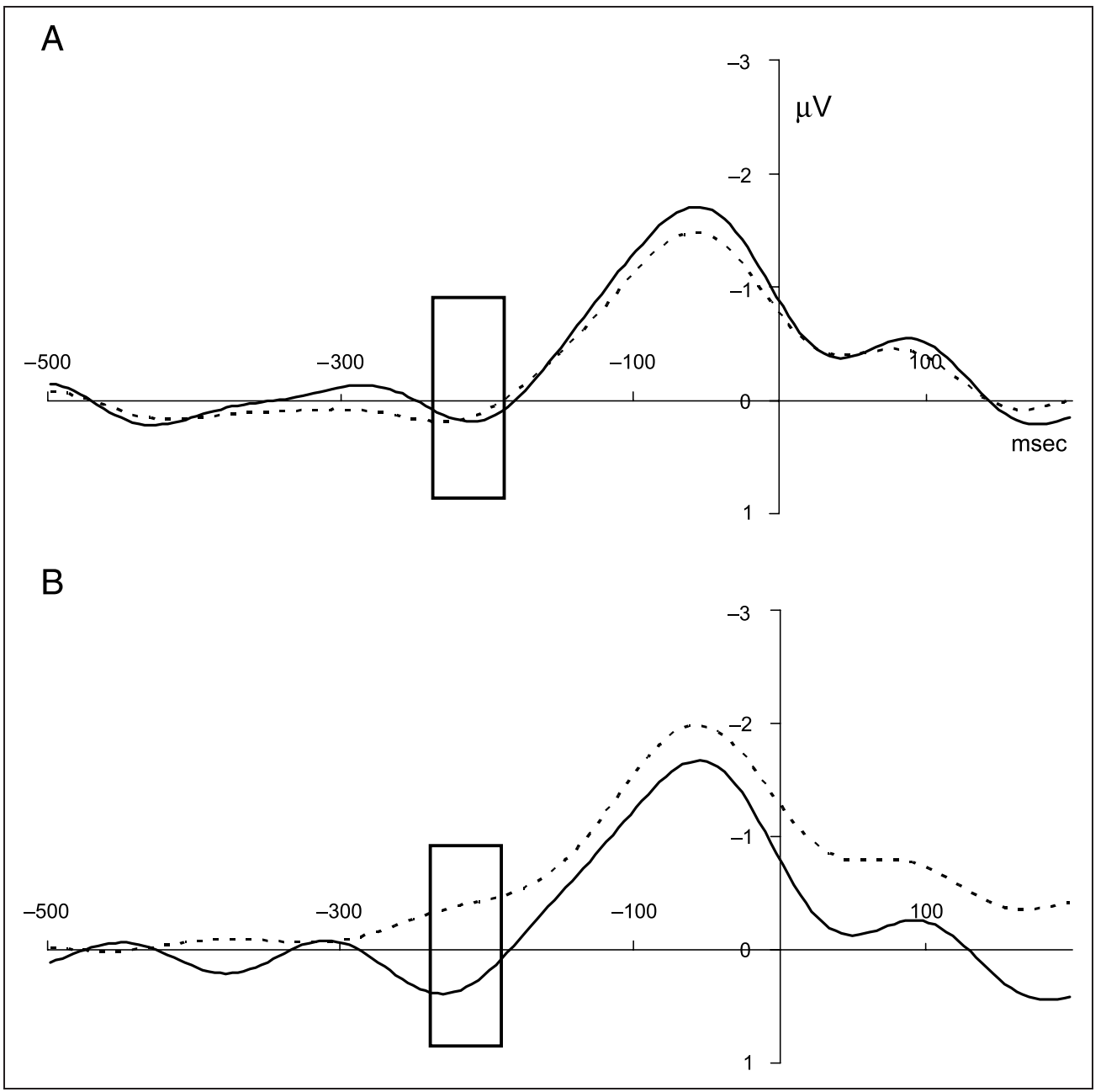

is equivocal. The reduction of the P300 in the incongruent condition (see Figures 5 and 6) suggests that some interaction occurs at this "early" level (starting $300 \mathrm{msec}$ poststimulus). Yet, the conflict is not fully resolved at this stage, as indicated by the LRP and LBR effects. In the high load condition, however, this trace of processing of the irrelevant dimension by the motor system is missing (see Figure 7). This supports the assumption of a common representation for numbers and physical size that is subserved by a shared neuronal locus, probably in the IPS (Cohen Kadosh \& Henik, 2006a, 2006b; Kaufmann et al., 2005; Pinel et al., 2004; Fias et al., 2003; Walsh, 2003; Schwarz \& Heinze, 1998). Why is this common representation only evident in the high load condition? Functional imaging showed that numerical comparison for small distances (the high load condition of the present study) recruits a larger part of the parietal cortex than for large distances (Cohen Kadosh et al., 2005). In the current experiment, the high load condition might have exceeded the capacity of the strictly number-specific parts of the parietal cortex and recruited areas that are also required for physical size comparison (see Berger, Henik, \& Rafal, 2005, for a similar idea in the field of spatial attention).

\section{Parallel and Interacting Streams of Processing}

The congruity effect found for both P300 and LRP in the low cognitive load condition suggests that the SCE manifests itself by a continuous flow of information processing, similar to other interference tasks (Gratton et al., 1988; Coles, Gratton, Bashore, Eriksen, \& Donchin, 1985). Hence, the conflict arises due to competition of parallel and interacting streams along the entire processing pathway, with its resolution occurring through interactions at both comparison and response-related stages. Note, however, that such an interpretation stands in contrast to the evidence for an additive, serial processing of numerical comparison from ERP, fMRI, and behavioral studies (Pinel, Dehaene, Rivière, \& LeBihan, 2001; Schwarz \& Ischebeck, 2000; Dehaene, 1996). It might be the case that the current conflict task is more sensitive to detect such deviation from additivity than one-dimensional comparison tasks. In any case, the 
assumption of a continuous flow of information would not weaken our conclusion that both physical and numerical dimensions are processed, at least partly, by distinct comparison mechanisms in the low cognitive load condition.

The finding of congruity effects both for the P300 and the LRP in the low cognitive load condition supports this idea because it points to interactions both in areas for response selection (LRP) and stimulus evaluation (P300). In fact, the conflict might be resolved in the IPS due to backward projections from the motor cortex. In the case presented here, when both dimensions compete for different responses in the motor cortex, selection is remitted to the IPS. The IPS, in addition to its prominent role in magnitude processing, is also involved in response selection and selection of taskrelevant information (Brass \& von Cramon, 2004; Göbel et al., 2004; Jiang \& Kanwisher, 2003; Bunge, Hazeltine, Scanlon, Rosen, \& Gabrieli, 2002). These processing stages are particularly crucial in solving conflicts. Furthermore, the role of the IPS in the final stage of conflict resolution in the present study is supported by the significant correlation between the P300 morphology and the behavioral data, and the observed temporal overlap between P300 and RTs in the incongruent condition. The P300 component serves as an indication of stimulus evaluation (Kok, 2001), and is partly generated in the IPS (Bledowski et al., 2004). Consequently, the sustained P300 activity in the incongruent condition indicates that the evaluation process was not complete at the time of the LRP occurrence. Hence, our data support the assumption that interaction between motor and parietal areas mediated the computations necessary to resolve the conflict and make an unambiguous response to the relevant dimension.

The ERP results from Experiment 2 complement the fMRI results from Experiment 1 . They indicate that the simultaneous SCE in the IPS and M1 might have been due to recurrent information processing between the P300 and the LRP. However, the ERP experiment solved the apparent contradiction between Experiment 1, which supports the notion of distinct mechanisms for numerical and physical processing, and previous experiments supporting a shared mechanism (Kaufmann et al., 2005; Pinel et al., 2004; Schwarz \& Heinze, 1998), by showing that both distinct and shared processing are possible, depending on the task demands.

\section{Conclusions}

We present a possible solution to the long-standing debate on number processing in the human brain (Cohen Kadosh \& Henik, 2006a, 2006b; Cohen Kadosh et al., 2005; Shuman \& Kanwisher, 2004; Fias et al., 2003; Walsh, 2003; Simon, 1999; Spelke \& Dehaene, 1999; Schwarz \& Heinze, 1998). Our results indicate that both shared and distinct processing models of magnitude are plausible and can be assessed with noninvasive neurophysiology and neuroimaging. However, which processing mechanism is employed depends on the task requirements and, more specifically, on the cognitive load. The specific task requirements further determine whether the resolution of conflicts between two dimensions will take place at an early or late processing stage. We show that in the case of low cognitive load, the conflict is processed up until the response selection stage. In the case of high cognitive load, however, the conflict between the relevant and irrelevant dimensions is resolved at an earlier stage, probably in the IPS.

\section{METHODS}

\section{Participants}

Fourteen participants ( 5 men, 1 left-handed, mean age $=$ 25.6 years, $S D=2.3$ years) in the fMRI experiment, and 14 participants $(7$ men, mean age $=25.82, S D=$ 3.94, all right-handed) in the ERP experiment. Participants were recruited from an academic environment and gave written informed consent. The study was approved by the local ethics committee. None of the participants reported significant neurological or psychiatric disorders.

\section{Stimuli}

Stimuli consisted of two digits that appeared at the center of a screen. Numerical and size comparisons were performed in separate blocks. Three types of pairs were used: congruent, neutral, and incongruent. In a congruent pair, the numerically larger digit was also physically larger (e.g., 2 4). In a neutral pair, the digits differed only in the relevant dimension (e.g., 24 for numerical comparison, 22 for size comparison). In an incongruent pair, the numerically larger digit was physically smaller (e.g., 2 4). The digits 1 to 9 were used, excluding the digit 5 .

\section{Procedure}

Participants were asked to decide which of two stimuli in a given display was numerically (numerical comparison) or physically (size comparison) larger. Each participant took part in two runs. Each run was composed of one block of numerical comparisons and one block of size comparisons. Participants were asked to respond as quickly as possible but to avoid errors and to attend only to the relevant dimension. They indicated their choices by pressing one of two keys corresponding to the side of the display with the selected digit (i.e., right hand for the right side, left hand for the left side). The order of the blocks was counterbalanced by an ABBA design. The stimuli were randomly sampled, and cor- 
rect responses were made equally often with the left and right hands.

\section{Experiment 1: fMRI Experiment}

We used Experimental Runtime System (Berisoft, Germany) running on a PC, as stimulus presentation software. The experiment was preceded by a training session, which allowed participants to complete as many trials as necessary to familiarize themselves with the task. During scanning, the computer display was projected onto a mirror mounted on the head coil. Participants' responses were registered by a fiber-optic response box (Current Designs, Philadelphia, USA).

Each trial began with an asterisk as a central fixation point, presented for $500 \mathrm{msec}$ at the center of a computer screen. Five hundred milliseconds after the fixation point disappeared, a pair of digits appeared for $1 \mathrm{sec}$. The intertrial interval was $8 \mathrm{sec}$. The interblock interval was $24 \mathrm{sec}$.

\section{Design}

The variables manipulated were: comparison (numerical, size) and congruency (congruent, neutral, or congruent). Thus, we had a $2 \times 3$ factorial design, with all variables manipulated within subjects.

\section{fMRI Scanning and Analysis}

Whole-brain fMRI data were acquired with a Siemens 1.5-T Magnetom Vision MRI scanner using a gradient-echo echo-planar imaging sequence (16 axial slices; repetition time $/$ echo time $=2000 / 60 \mathrm{msec}$; flip angle $=90^{\circ}$, field of view $=210 \times 210 \mathrm{~mm}$, voxel size: $3.28 \times 3.28 \times 5 \mathrm{~mm}$ ) Each run comprised the acquisition of 390 volumes and contained 72 trials ( 12 trials $\times 2$ tasks $\times 3$ congruency). Stimulus presentation was synchronized with the fMRI sequence at the beginning of each trial. Each scanning session included the acquisition of a high-resolution T1weighted three-dimensional volume (voxel dimensions $=$ $1 \times 1 \times 1 \mathrm{~mm}$ ) for coregistration and anatomical localization of functional data. Data were preprocessed and analyzed using the BrainVoyager 4.9 software package. Statistical analysis was based on the cortex-based general linear model (GLM) of the experiment (Cohen Kadosh et al., 2005; Munk et al., 2002). The first four volumes of each run were discarded to allow for T1 equilibration. 3-D motion correction and Talairach transformation (Talairach \& Tournoux, 1988) were performed for the remaining set of functional data of each participant. The 3-D functional dataset was resampled to a voxel size of $3 \times 3 \times 3 \mathrm{~mm}$, spatial smoothing with a Gaussian kernel (full width at half maximum $=8 \mathrm{~mm}$ ), linear trend removal, temporal high-pass filtering (high pass: $0.00647 \mathrm{~Hz}$ ), and autocorrelation removal. For the
GLM, each of the six conditions of the experimental design (numerical comparison: congruent, incongruent, neutral; size comparison: congruent, incongruent, neutral) was defined as a predictor that assumed the value of 1 for the volume during which a pair of stimuli belonging to that condition was presented and the following volume, and 0 for all other volumes. Error trials were modeled separately. The remaining volumes served as baseline. The cortex-based GLM of the experiment, with predictors convolved with a hemodynamic response function (Boynton, Engel, Glover, \& Heeger, 1996), was computed from the $28 z$-normalized volume time courses. The cortex-based GLM approach (Goebel \& Singer, 1999) was developed for fMRI studies whose hypotheses only pertained to cortical areas. In these cases, data analysis can be confined to cortical voxels, avoiding unnecessarily strict Bonferroni correction. The cortex mask used here was derived from the cortex reconstruction of the Montreal Neurological Institute (MNI) template brain, transformed into Talairach space, and contained 23,848 voxels. A random effects analysis was employed. Effects are only shown if the associated $p$ value yielded $p^{\prime}<.05$ (corrected for multiple comparisons). For the significant voxels selected for both comparison tasks across congruity conditions, we performed a two-way ANOVA (Statistica 6.0) on the beta weights, with comparison (size and numerical) and congruency (congruent, neutral and incongruent) as factors. The voxels that showed a significant effect for the neutral conditions were deemed to be activated by a given dimension (i.e., physical size or numerical value) without the contamination of the irrelevant dimension. These voxels were also the basis for the selection of the motor cortex ROI.

\section{Motor Cortex ROI}

For the analysis of the irrelevant hand movement, we defined the motor cortex as an ROI according to the comparison between the right- and left-hand response in the neutral conditions $\left(t\right.$ test, fixed effects, $p^{\prime}<.05$, right-hand center of mass 39, - 25, 52, 8287 voxels, lefthand center of mass $-37,-26,52,4859$ voxels). We used the neutral conditions in order to keep the results of the ROI congruity contrasts orthogonal and not bias the ROI selection toward the incongruent condition. Individual movements activated similar M1 areas around the knob defining the hand region (Yousry et al., 1997) (see Figure 3). We performed an ANOVA over the ROI time courses with the following parameters: hemisphere (right, left), comparison (numerical, size), congruency (congruent, neutral or incongruent), and response hand (response, nonresponse irrelevant). Thus, we had a $2 \times$ $2 \times 3 \times 2$ factorial design, with all variables within subjects.

Similarly to the LRP (see below), the LBR can be obtained by subtracting ipsilateral activity (nonresponse 
hand) from contralateral activity (response hand) in the M1. LBR was calculated using the following equation:

$$
\begin{aligned}
\text { LBR }= & \frac{(\text { Left hand }- \text { Right Hand })_{\text {right M1 }}}{2} \\
& +\frac{(\text { Right hand }- \text { Left Hand })_{\text {left M1 }}}{2}
\end{aligned}
$$

If the irrelevant dimension is processed throughout response initiation, the LBR for the conflict condition (i.e., incongruent in the current study) will yield less activation than the no-conflict condition (i.e., neutral and congruent in the current study).

\section{Experiment 2: ERP Experiment}

In addition to the congruency conditions in the fMRI experiment, in this experiment, we comprised the digits that we used in the fMRI experiment into two numerical distances in order to manipulate the cognitive load: high cognitive load (numerical distance 1, digit pairs: 1-2, 3-4, 6-7, or 8-9), or low cognitive load (numerical distance 5, digit pairs: 1-6, 2-7, 3-8, or 4-9). Each number was presented an equal number of times in each load condition.

\section{Procedure}

Stimulus onset asynchrony (SOA) was 900 to $1100 \mathrm{msec}$, with an average of $1000 \mathrm{msec}$, and stimulus presentation time was $1,000 \mathrm{msec}$. The presentation of the experiment and the collection of the behavioral data were controlled by a personal computer using e-prime (Psychology Software Tools, Pittsburgh, USA).

\section{Design}

The variables manipulated were: comparison (numerical, size), congruity (incongruent, neutral or congruent), and cognitive load (low, high). Thus, we had a $2 \times 3 \times 2$ factorial design, with all variables manipulated within subjects. The actual measurements were preceded by a short training session of 24 trials. An experimental block (numerical or size comparisons) consisted of 144 trials and lasted approximately $10 \mathrm{~min}$. Each participant took part in four blocks. Thus, there were 48 trials for each condition. The order of the blocks was presented in an ABBA design for half of the participants and a BAAB design for the other half.

\section{Electroencephalography Recording and Analysis}

Electroencephalographic (EEG) data were gathered using a 128-Ag/AgCl electrode Geodesic Sensor Net (Electri- cal Geodesics, Oregon, USA) with 16-bit digitizing at $250 \mathrm{~Hz}$. During recording, all channels were referenced to the $\mathrm{Cz}$ electrode and band-pass filtered 0.1 to $100 \mathrm{~Hz}$. Throughout measurements, impedances were kept below $40 \mathrm{k} \Omega$. EEG data preprocessing and analysis were performed using the Netstation platform 4.1 (Electrical Geodesics).

The EEG data analysis included only trials with correct responses. For the ERP analysis, raw data were digitally low-pass filtered at $40 \mathrm{~Hz}$ (passband gain: $-0.1 \mathrm{~dB}$; stopband gain: $-40.0 \mathrm{~dB}$; rolloff: $2.0 \mathrm{~Hz}$ ) and segmented into epochs from $-200 \mathrm{msec}$ prestimulus presentation until 800 msec after stimulus onset. Segments with activity exceeding $\pm 70 \mu \mathrm{V}$ in any channel were excluded from further analysis. Not more than 25\% of trials were discarded due to artifacts (Picton et al., 2000). Bad channels were replaced using spherical splines. Data were averaged and re-referenced to an average electrode. The averages were then baseline corrected, with the 100-msec prestimulus period serving as baseline. Further statistical analysis focused on the P300 component. The P300 was defined as the largest positive deflection within a predefined time window (300500 msec poststimulus onset). Mean area amplitudes were calculated on individual averages at the Pz electrode (analogous to electrode 68 in the EGI system) and assessed with repeated measure ANOVA with the same factorial design as described above.

In contrast to the stimulus-locked ERP analysis, for the LRP analysis the trials were locked to the response. When investigating response-related processes, it has been shown to be more useful to look at the responselocked LRP rather than the stimulus-locked LRP, due to increased latency jitter in the latter (Gevers, Ratinckx, De Baene, \& Fias, 2006; Keus, Jenks, \& Schwarz, 2005; Masaki, Takasawa, \& Yamazaki, 2000). Hence, the differences between the congruency conditions will be more clearly visible under response-locked analysis. For the LRP analysis, raw data were digitally low-pass filtered at $8 \mathrm{~Hz}$ (passband gain: $-0.1 \mathrm{~dB}$; stopband gain $-40.0 \mathrm{~dB}$; rolloff: $2.0 \mathrm{~Hz}$ ) and segmented for each hand separately into epochs starting $-500 \mathrm{msec}$ before the response, and lasting until 200 msec after the response. Segments were inspected for artifacts, and trials with activity exceeding $\pm 70 \mu \mathrm{V}$ in any channel were excluded from further analysis. Not more than $25 \%$ of trials were discarded due to artifacts. Data were averaged and baseline corrected, with the -500 to $-450 \mathrm{msec}$ interval before response serving as baseline. LRP grand averages were calculated using the following equation (Rugg \& Coles, 1996):

$$
\begin{aligned}
\mathrm{LRP}= & \frac{\text { Mean }(\mathrm{C} 4-\mathrm{C} 3)_{\text {left-hand response }}}{2} \\
& +\frac{\text { Mean }(\mathrm{C} 3-\mathrm{C} 4)_{\text {right-hand response }}}{2}
\end{aligned}
$$


Mean area amplitude analysis was performed on the grand-average waves within the time window of -240 to $-200 \mathrm{msec}$ prior to response. The time window was chosen from visual inspection of the grand-average waves. Because both congruent and neutral conditions were not expected to induce motor activation ipsilateral to the response hand (i.e., no conflict occurs), the two conditions were collapsed and compared to the incongruent condition (i.e., conflict occurs). This, in turn, allowed us to increase the reliability and the signal-tonoise ratio of the congruity effect.

\section{Acknowledgments}

This work was supported by the Kreitman Foundation, Minerva Seed Grant by the Max Planck Society, Israel Foundations Trustees (R. C. K.), and Minerva short-term grant by the Max Planck Society (K. C. K.). The fMRI experiments were conducted at the Brain Imaging Center Frankfurt am Main, supported by the German Ministry for Education and Research (BMBF; DLR 01GO0203).

We thank Harald Mohr for programming the fMRI experiment. We also thank Joseph Tzelgov and Jan Lammertyn for their helpful suggestions for the fMRI experiment.

The fMRI and the ERP experiments were initially submitted as two separate manuscripts, reporting the fMRI (R. C. K., H. A., K. C. K., and D. E. J. L.) and ERP (R. C. K., K. C. K., D. E. J. L., W. G., A. B., \& A. H., where R. C. K. and K. C. K. equally contributed to the ERP manuscript)

The authors declare that they do not have any conflict of interest.

Reprint requests should be sent to Roi Cohen Kadosh, Institute of Cognitive Neuroscience, University College London, London, UK, or via e-mail: r.cohenkadosh@ucl.ac.uk.

\section{REFERENCES}

Berger, A., Henik, A., \& Rafal, R. (2005). Competition between endogenous and exogenous orienting of visual attention.

Journal of Experimental Psychology: General, 134, 207-221.

Bledowski, C., Cohen Kadosh, K., Wibral, M., Rahm, B., Bittner, R. A., Hoechstetter, K., et al. (2006). Mental chronometry of working memory retrieval: A combined functional magnetic resonance imaging and event-related potentials approach. Journal of Neuroscience, 26, 821-829.

Bledowski, C., Prvulovic, D., Hoechstetter, K., Scherg, M., Wibral, M., Goebel, R., et al. (2004). Localizing P300 generators in visual target and distractor processing: A combined event-related potentials and functional magnetic resonance imaging study. Journal of Neuroscience, 24, 9353-9360.

Boynton, G. M., Engel, S. A., Glover, G. H., \& Heeger, D. J. (1996). Linear systems analysis of functional magnetic resonance imaging in human V1. Journal of Neuroscience, 16, 4207-4221.

Brass, M., \& von Cramon, D. Y. (2004). Selection for cognitive control: A functional magnetic resonance imaging study on the selection of task-relevant information. Journal of Neuroscience, 24, 8847-8852.

Bunge, S. A., Hazeltine, E., Scanlon, M. D., Rosen, A. C., \& Gabrieli, J. D. (2002). Dissociable contributions of prefrontal and parietal cortices to response selection. Neuroimage, 17, 1562-1571.

Bush, G., Luu, P., \& Posner, M. I. (2000). Cognitive and emotional influences in anterior cingulate cortex. Trends in Cognitive Sciences, 4, 215-222.

Cohen Kadosh, R., \& Henik, A. (2006a). A common representation for semantic and physical properties: A cognitive-anatomical approach. Experimental Psychology, 53, 87-94.

Cohen Kadosh, R., \& Henik, A. (2006b). When a line is a number: Color yields magnitude information in a digit-color synesthete. Neuroscience, 137, 3-5.

Cohen Kadosh, R., Henik, A., \& Rubinsten, O. (2007). The effect of orientation on number word processing. Acta Psychologica, 124, 370-381.

Cohen Kadosh, R., Henik, A., Rubinsten, O., Mohr, H., Dori, H., Van de Ven, V., et al. (2005). Are numbers special? The comparison systems of the human brain investigated by fMRI. Neuropsychologia, 43, 1238-1248.

Coles, M. G. H., Gratton, G., Bashore, T. R., Eriksen, C. W., \& Donchin, E. (1985). A psychophysiological investigation of the continuous flow model of human information processing. Journal of Experimental Psychology: Human Perception and Performance, 11, 529-553.

Dehaene, S. (1996). The organization of brain activations in number comparison: Event-related potentials and the additive-factors method. Journal of Cognitive Neuroscience, 8, 47-68.

Dehaene, S. (2003). The neural basis of the Weber-Fechner law: A logarithmic mental number line. Trends in Cognitive Sciences, 7, 145-147.

Dehaene, S., \& Akhavein, R. (1995). Attention, automaticity, and levels of representation in number processing. Journal of Experimental Psychology: Learning, Memory, and Cognition, 21, 314-326.

Dehaene, S., Naccache, L., Le Clec'H, G., Koechlin, E., Mueller, M., Dehaene-Lambertz, G., et al. (1998). Imaging unconscious semantic priming. Nature, 395, 597-600.

Dehaene, S., Piazza, M., Pinel, P., \& Cohen, L. (2003). Three parietal circuits for number processing. Cognitive Neuropsychology, 20, 487-506.

Fias, W., Lammertyn, J., Reynvoet, B., Dupont, P., \& Orban, G. A. (2003). Parietal representation of symbolic and nonsymbolic magnitude. Journal of Cognitive Neuroscience, 15, 1-11.

Gevers, W., Ratinckx, E., De Baene, W., \& Fias, W. (2006). Further evidence that the SNARC effect is processed along a dual-route architecture: Evidence from the lateralized readiness potential. Experimental Psychology, 53, 58-68.

Göbel, S. M., Johansen-Berg, H., Behrens, T., \& Rushworth, M. F. S. (2004). Response-selection-related parietal activation during number comparison. Journal of Cognitive Neuroscience, 16, 1536-1551.

Goebel, R., \& Singer, W. (1999). Cortical surface-based statistical analysis of functional magnetic resonance imaging data. Neuroimage, 9, S64.

Gratton, G., Coles, M. G. H., Sirevaag, E. J., Eriksen, C. W., \& Donchin, E. (1988). Pre- and poststimulus activation of response channels: A psychophysiological analysis. Journal of Experimental Psychology: Human Perception and Performance, 14, 331-344.

Henik, A., \& Tzelgov, J. (1982). Is three greater than five: The relation between physical and semantic size in comparison tasks. Memory \& Cognition, 10, 389-395.

Jiang, Y., \& Kanwisher, N. (2003). Common neural substrates for response selection across modalities and mapping paradigms. Journal of Cognitive Neuroscience, 15, 1080-1094. 
Kaufmann, L., Koppelstaetter, F., Delazer, M., Siedentopf, C., Rhomberg, P., Golaszewski, S., et al. (2005). Neural correlates of distance and congruity effects in a numerical Stroop task: An event-related fMRI study. Neuroimage, 25, 888-898

Kerns, J. G., Cohen, J. D., MacDonald, A. W., Cho, R. Y., Strenger, V. A., \& Carter, C. S. (2004). Anterior cingulate conflict monitoring and adjustments in control. Science, 303, 1023.

Keus, I. M., Jenks, K. M., \& Schwarz, W. (2005). Psychophysiological evidence that the SNARC effect has its functional locus in a response selection stage. Cognitive Brain Research, 24, 48-56.

Kok, A. (2001). On the utility of P3 amplitude as a measure of processing capacity. Psychophysiology, 38, 557-577.

Lavie, N. (2005). Distracted and confused?: Selective attention under load. Trends in Cognitive Sciences, 9, 75-82.

Lavie, N., Hirst, A., de Fockert, J. W., \& Viding, E. (2004). Load theory of selective attention and cognitive control. Journal of Experimental Psychology: General, 133, 339-354.

Lavie, N., \& Tsal, Y. (1994). Perceptual load as a major determinant of the locus of selection in visual attention. Perception \& Psychophysics, 56, 183-197.

Linden, D. E. J. (2005). The P300: Where in the brain is it produced and what does it tell us? The Neuroscientist, 11, 563-576

Masaki, H., Takasawa, N., \& Yamazaki, K. (2000). An electrophysiological study of the locus of the interference effect in a stimulus-response compatibility paradigm. Psychophysiology, 37, 464-472.

Moyer, R. S., \& Landauer, T. K. (1967). Time required for judgment of numerical inequality. Nature, 215, 1519-1520.

Munk, M. H. J., Linden, D. E. J., Muckli, L., Lanfermann, H., Zanella, F. E., Singer, W., et al. (2002). Distributed cortical systems in visual short-term memory revealed by event-related functional magnetic resonance imaging. Cerebral Cortex, 12, 866-876.

Nieder, A. (2004). The number domain-Can we count on parietal cortex? Neuron, 44, 407-409.

Picton, T. W., Bentin, S., Berg, P., Donchin, E., Hillyard, S. A., Johnson, R., et al. (2000). Guidelines for using human event-related potentials to study cognition: Recording standards and publication criteria. Psychophysiology, 37, 127-152.

Pinel, P., Dehaene, S., Rivière, D., \& LeBihan, D. (2001). Modulation of parietal activation by semantic distance in a number comparison task. Neuroimage, 14, 1013-1026.

Pinel, P., Piazza, M., Le Bihan, D., \& Dehaene, S. (2004). Distributed and overlapping cerebral representations of number, size, and luminance during comparative judgments. Neuron, 41, 983-993.

Price, C. J., \& Friston, K. J. (2002). Degeneracy and cognitive anatomy. Trends in Cognitive Sciences, 6, 416-421.
Rees, G., Frith, C. D., \& Lavie, N. (1997). Modulating irrelevant motion perception by varying attentional load in an unrelated task. Science, 278, 1616-1619.

Rugg, M. D., \& Coles, M. G. H. (1996). Electrophysiology of mind: Event-related brain potentials and cognition. Oxford: Oxford University Press.

Schwartz, S., Vuilleumier, P., Hutton, C., Maravita, A., Dolan, R. J., \& Driver, J. (2005). Attentional load and sensory competition in human vision: Modulation of fMRI responses by load at fixation during task-irrelevant stimulation in the peripheral visual field. Cerebral Cortex, 15, 770-786.

Schwarz, W., \& Heinze, H. J. (1998). On the interaction of numerical and size information in digit comparison: A behavioral and event-related potential study. Neuropsychologia, 36, 1167-1179.

Schwarz, W., \& Ischebeck, A. (2000). Sequential effects in number comparison. Journal of Experimental Psychology: Human Perception and Performance, 26, 1606-1621.

Schwarz, W., \& Ischebeck, A. (2003). On the relative speed account of the number-size interference in comparative judgment of numerals. Journal of Experimental Psychology: Human Perception and Performance, 29, 507-522.

Shuman, M., \& Kanwisher, N. (2004). Numerical magnitude in the human parietal lobe: Tests of representational generality and domain specificity. Neuron, 44, 557-569.

Simon, T. J. (1999). The foundations of numerical thinking in a brain without numbers. Trends in Cognitive Sciences, 3, 363-365.

Spelke, E., \& Dehaene, S. (1999). Biological foundations of numerical thinking. Trends in Cognitive Sciences, 3, 365-366.

Stephan, K. E., Marshall, J. C., Friston, K. J., Rowe, J. B., Ritzal, A., Zilles, K., et al. (2003). Lateralized cognitive processes and lateralized task control in the human brain. Science, 301, 384-386.

Talairach, J., \& Tournoux, P. (1988). Co-planar stereotaxic atlas of the buman brain. New York: Thieme.

Tzelgov, J., Meyer, J., \& Henik, A. (1992). Automatic and intentional processing of numerical information. Journal of Experimental Psychology: Learning, Memory, and Cognition, 18, 166-179.

Walsh, V. (2003). A theory of magnitude: Common cortical metrics of time, space and quantity. Trends in Cognitive Sciences, 7, 483-488.

Weissman, D. H., Warner, L. M., \& Woldorff, M. G. (2004). The neural mechanisms for minimizing cross-modal distraction. Journal of Neuroscience, 28, 10941-10949.

Wojciulik, E., \& Kanwisher, N. (1999). The generality of parietal involvement in visual attention. Neuron, 23, 747-764.

Yousry, T. A., Schmid, U. D., Alkadhi, H., Schmidt, D., Peraud, A., Buettner, A., et al. (1997). Localization of the motor hand area to a knob on the precentral gyrus. A new landmark. Brain, 120, 141-157. 